\title{
RESEARCH INTO CONNECTION BETWEEN SPECTRAL RESONANCE STRUCTURES AND HARMONICS OF IONOSPHERIC ALFVÉN RESONATOR
}

\author{
K. Prikner ${ }^{\dagger}$ \\ Institute of Geophysics CAS, Prague, Czech Republic \\ F.Z. Feygin \\ O.Yu. Shmidt Institute of Physics of Earth RAS \\ Moscow, Russia,feygin@ifz.ru
}

\author{
Yu.G. Khabazin \\ O.Yu. Shmidt Institute of Physics of Earth RAS \\ Moscow, Russia, khabazin@ifz.ru
}

\begin{abstract}
We present the results of modeling of the ionospheric Alfvén resonator (IAR) and compare the results of electromagnetic waves passing through IAR to Earth's surface with the dynamic spectrum of simultaneous observations of spectral resonance structures (SRS). IAR is simulated using ionospheric parameters obtained from measurements made with the CP-1 program of the Scandinavian EISCAT radar. The IAR model is employed to calculate coefficients of reflection $R C(f)$ and transmission $T C(f)$ of electromagnetic waves in the frequency range $0-5 \mathrm{~Hz}$. The observed dynamic
\end{abstract}

SRS spectrograms consist of spectral lines, in which frequencies, time variations of frequencies, and distances between adjacent resonant lines are confidently determined. The calculated frequencies of maxima of the signal transmission coefficient TC to Earth's surface correspond to the observed frequencies of the dynamic spectrum of SRS.

Keywords: ionospheric Alfvén resonator, reflection and transmission coefficients, dynamic spectra, spectral resonance structures.

\section{INTRODUCTION}

Numerous studies of spectral resonance structures (SRS) attribute their occurrence in Earth to the effect the ionospheric Alfvén resonator (IAR) [Pokhotelov et al., 2001] exerts on Alfvén wave propagation through Earth's ionosphere in a frequency range from a fraction of hertz to a few hertz. The first experimental studies of SRS were carried out at the mid-latitude station near Nizhny Novgorod and at the high-latitude station in Kilpisjärvi, Finland ( $L=6)$ ) [Belyaev et al., 1989, 1990]. Typical SRS are shown in Figure 1 [Pokhotelov et al., 2003].

SRS were examined at high latitudes [Yahnin et al., 2003; Pokhotelov et al., 2003]. Guglielmi and Potapov [2017] have shown that the occurrence of SRS at Mondy Observatory is connected with the solar wind. In addition to ground-based observations, space-based observations of SRS were made using the FREJA and FAST satellites [Grzesiak, 2000; Chaston et al., 2002]. When studying the connection of SRS on Earth's surface with the IAR effect, an ionospheric model was constructed from known numerical ionospheric models and real ionospheric parameters obtained with ionosondes [Belyaev et al., 1999; Semenova, Yahnin, 2014]. We have used real data on ionospheric plasma to construct the ionospheric model. The data were obtained by application of the CP-1 program of the Scandinavian EISCAT radar, located near the high-latitude station in Kilpisjärvi, which observed SRS. The purpose of our study is to quantitatively describe SRS spectra occurring during the passage of electromagnetic waves through the ionosphere to Earth's surface in the frequency range $0-5 \mathrm{~Hz}$ under the action of the Alfvén resonator.

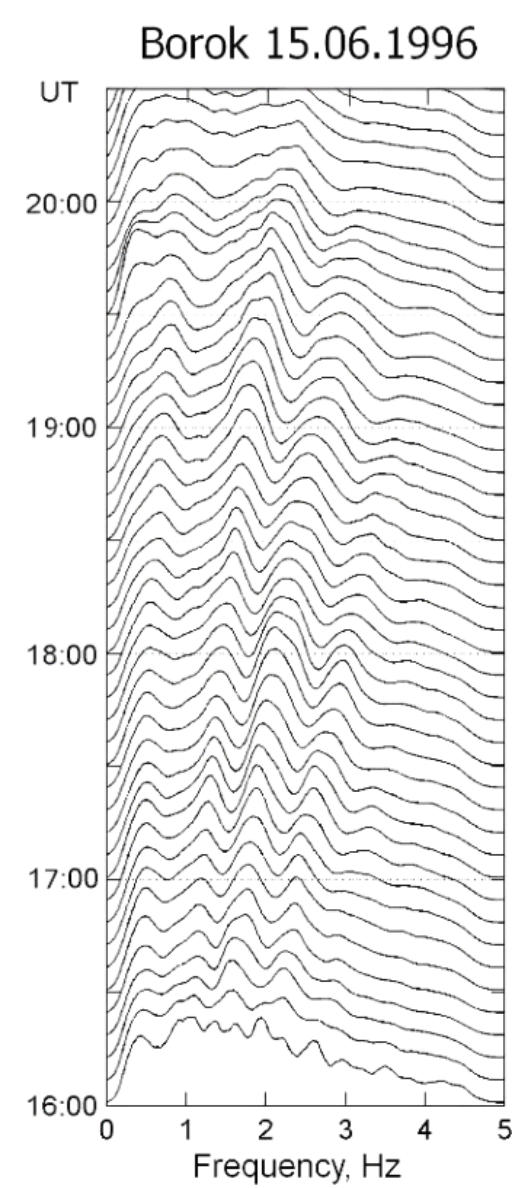

Figure 1. Typical dynamic SRS spectrum recorded at Borok Observatory on September 15, 1996.

This paper is dedicated to the memory of the famous Czech geophysicist Dr. Karel Prikner. This paper is based on unpublished results obtained by K. Prikner together with co-authors. 


\section{Application of the method of numerical simulation of ionospheric signal filtering based on vertical sounding data from the Scandinavian EISCAT radar}

To study the SRS dynamic spectrum, we apply the numerical simulation method described in detail by Prikner, Vagner [1983, 1991]. This method was also used to explain the formation of spectra of geomagnetic pulsations [Prikner et al., 2000; Mursula et al., 2000]. For the analysis, we take the interval of SRS dynamic spectrum 19:30-20:30 UT on September 21, 1998, observed at the station in Kilpisjärvi $\Phi=69.02^{\circ} \mathrm{N}, \lambda=20.86^{\circ} \mathrm{E}, L=5.9$.

The interval of the dynamic spectrum is shown in Figure 2. For this interval there are measurements of the vertical profile of ionospheric plasma parameters obtained using the CP-1 program of the EISCAT radar. The numerical simulation method in hand allows us to obtain values of IAR signal frequencies and values of $\mathbf{E}$ and $\mathbf{B}$ electric and magnetic wave fields both on Earth's surface and at any height level of IAR. To simulate wave propagation in IAR, we have chosen a homogeneous flat Alfvén wave of the frequency $f$ with the wave vector $\mathbf{k}$, incident on an ionospheric stratified magnetically active medium with an external geomagnetic field $\mathbf{B}_{E}$. It is assumed that $\mathbf{k} \| \mathbf{B}_{E}$. With this propagation direction, the Alfvén wave is a left-handed polarized L-mode.

The main initial parameters for the simulation of ionospheric medium are the vertical profiles of the electron-ion density of the quasi-neutral ionosphere $N_{\mathrm{e}}(\mathrm{z})$ really observed by the EISCAT radar, the profiles of the effective ion mass of the total plasma content, and the profiles of effective ion and electron collisions [Prikner et al., 2001].

During the simulation, we calculate amplitudes of the full wave field (E, B). Hence, we determine transmission and reflection coefficients, which are important characteristics of IAR.

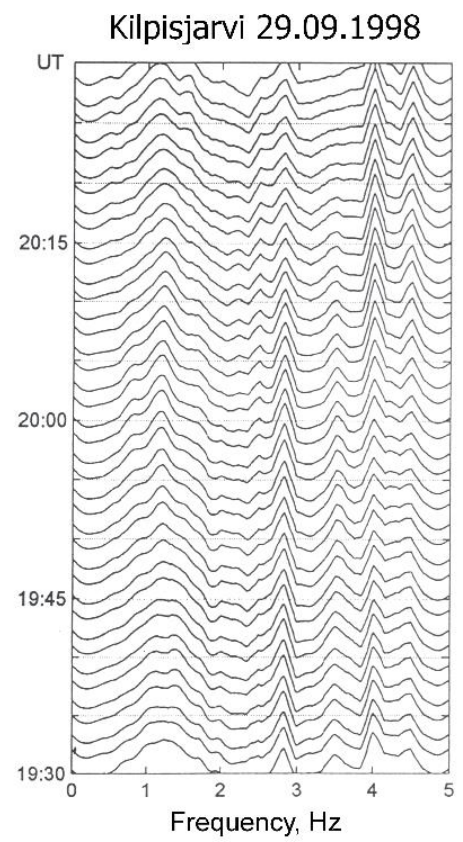

Figure 2. Interval of the SRS dynamic spectrum 19.30 20.30 UT on September 21, 1998, observed at the highlatitude station in Kilpisjärvi.
The transmission coefficient (TC) at $f$ near Earth's surface is found from horizontal amplitude components of the wave field $B$ :

$$
T C(f)=B_{h}(f, z=0) / B_{h}^{\text {inc }}\left(f, z_{\max }\right),
$$

where $B_{h}$ is the horizontal component of the wave arriving at the surface, $B_{h}^{\text {inc }}$ is the horizontal amplitude component of the wave incident on the ionosphere.

The reflection coefficient $R C$ on the upper boundary of the ionosphere $z=Z_{\max }$ (the assumed upper boundary of IAR)

$$
R C(f)=B_{h}^{\mathrm{refl}}\left(f, z_{\max }\right) / B_{h}^{\mathrm{inc}}\left(f, z_{\max }\right),
$$

where $B_{h}^{\text {refl }}$ is the horizontal amplitude component of the wave reflected to the magnetosphere at $z=z_{\max }$. For real IAR the upper boundary is defined by a steep gradient of Alfvén velocity $V_{\mathrm{A}}$ depending on ion density, and may be taken equal to $z_{\max }=2000 \mathrm{~km}$. From equations (1) and (2) it follows that $T C$ and $R C$ are horizontal amplitudes normalized to the incident wave amplitude.

The EISCAT CP-1 program measures vertical profiles of ionospheric plasma parameters over Troms $\emptyset$ (Norway, $\varphi=69.66^{\circ} \mathrm{N}, \lambda=18.95^{\circ} \mathrm{E}$ ) near the high-latitude station in Kilpisjärvi. These measurements were made only to $z_{1}=600 \mathrm{~km}$; therefore to construct IAR to $z_{\max }=2000 \mathrm{~km}$, the EISCAT CP-1 data are supplemented with adequate data from the IRI-95 model. According to this model, the decrease in the ionospheric plasma density is nearly exponential; therefore we take the density distribution approximation in the form of

$$
N_{\mathrm{e}}(\mathrm{z})=N_{\mathrm{e}}\left(\mathrm{z}_{1}\right) 10^{-a(\mathrm{z}-\mathrm{z} 1)}
$$
with $a=1.95 \cdot 10^{-3} \mathrm{~km}^{-1}$.

Figure 3, $a$ shows vertical profiles of ion (marked line) and electron (solid line) effective collision frequencies.
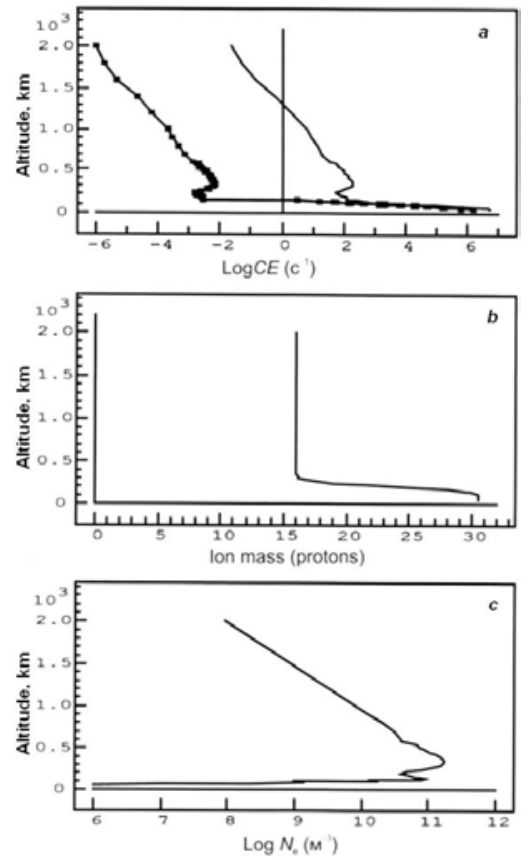

Figure 3 . Vertical profiles of ion (marked line) and electron (solid line) effective collision frequencies (a); effective ion mass in proton mass units $(b)$; electron-ion density (c) up to $z_{\max }=2000 \mathrm{~km}$ (Kilpisjärvi, 20:00 UT, September 21, 1998) 
From these profiles we calculate $T C(f)$ and $R C(f)$ in the $0-5 \mathrm{~Hz}$ frequency range. On the upper boundary of IAR at $z_{\max }=2000 \mathrm{~km}$ there might have been an Alfvén wave incidence on the ionosphere with the external magnetic field at an angle $I=77^{\circ}$ to the ionosphere, which corresponds to conditions of the high-latitude station in Kilpisjärvi. Figure $4, a, b$ presents the results of the numerical model frequency dependences of $R C(f)$ and $T C(f)$ respectively. These normalized curves of the horizontal amplitude demonstrate explicit series of harmonics above IAR's fundamental frequency $f_{0} \sim 0.3 \mathrm{~Hz} ; R C$ minima coincide with $T C$ maxima. Note that $R C<1$ for all frequencies, whereas $T C(f)$ can take any values because of the possible resonant amplification of signals in the ionospheric Alfvén resonator [Prikner, Vagner, 1990].

Figure 4 shows a series of higher harmonics at frequencies of $0.75,1.30,1.75,2.25,2.70,3.15,3.60,4.00$, and $4.45 \mathrm{~Hz}$. Most of them are depicted in Figure 2, which represents the dynamic SRS spectrum of a signal at the high-latitude station in Kilpisjärvi. Maxima of signal power spectra are at $0.7,1.2,1.7,2.4,2.8,3.3$, 3.7, 4.1, and $4.5 \mathrm{~Hz}$. They closely match SRS maxima, which are likely to be caused by the IAR effect on the initial noise electromagnetic signal propagating down to the ionosphere from remote magnetospheric regions.
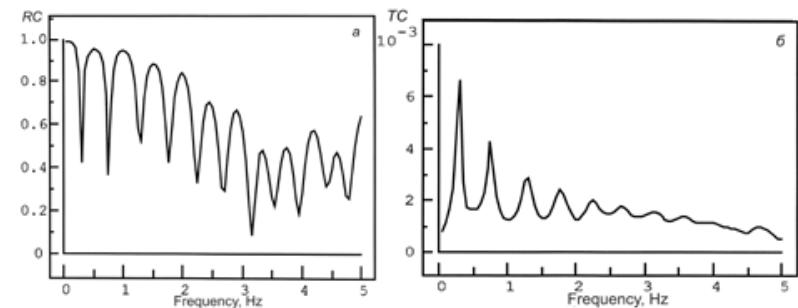

Figure 4. Frequency dependences of $R C(f)(a)$ and $T C(f)$ (b) at 20:00 UT on September 21, 1998

\section{CONCLUSION}

We have reported the results of the study of spectral resonance structures in the $0-5 \mathrm{~Hz}$ frequency range caused by the ionospheric Alfvén resonator. The close agreement between the results of numerical simulation of ionospheric filtration, obtained from EISCAT radar data, and simultaneous observations of SRS at the highlatitude station in Kilpisjärvi on September 21, 1998 allows us to conclude that the observed SRS are a direct consequence of the passage of magnetospheric electromagnetic noise in the $0-5 \mathrm{~Hz}$ frequency range through the filter of the ionospheric Alfvén resonator.

The work was supported by grant of the RAS Presidium Program No. 28 and by the state task of IPE RAS.

\section{REFERENCES}

Belyaev P.P., Polyakov S.V., Rapoport V.O., Trakhtengertz V.Y. Experimental studies of resonance structure in the spectrum of atmospheric electromagnetic background noise in the range of short-period geomagnetic pulsations. Radiophysics and Quantum Electronics. 1989, vol. 32, pp. 491-498.

Belyaev P.P., Polyakov S.V., Rapoport V.O., Trakhtengertz V.Y. The ionospheric Alfvén resonator. J. Atmos. Ter. Phys. 1990, vol. 52, pp. 781-788.

Belyaev P.P., Bosinger T., Isaev S.V., Kangas J. First evidence at high latitudes for the ionospheric Alfvén resonator. $J$. Geophys. Res. 1999, vol. 104, pp. 4305-4317.

Chaston C.C., Bonell J.W., Carlson C.V., Berthomier M, Peticolas L.M., Roth I., McFadden J.P. Electron acceleration in the ionospheric Alfvén resonator. J. Geophys. Res. 2002, vol. 107. DOI: 1029/2002JA009272.

Grzesiak M. Ionospheric Alfvén resonator. Geophys. Res. Let. 2000, vol. 27, pp. 923-926.

Guglielmi A.V., Potapov A.S. Interplanetary magnetic field effect on ULF oscillations of ionospheric resonator. Kosmicheskie issledovaniya [Cosmic Res.]. 2017, vol. 55, no. 4, pp. 263-267. (In Russian).

Mursula K., Prikner K., Feygin F.Z., Braysy T., Kangas J., Kerttula R., Pollari P., Pikkarainen T., Pokhotelov O.A. Nonstationary Alfvén resonator: new results on Pc1 pearls and IPDP events. J. Atm. Solar-Ter. Phys. 2000, vol. 62, pp. 299-309.

Pokhotelov O.A., Khruschev V., Parrot V., Senchenkov S., Pavlenko V.P. Ionospheric Alfvén resonator revisited. Feedback instability. J. Geophys. Res. 2001, vol. 106, pp. 25813-25824.

Pokhotelov O.A., Feygin F.Z, Khabazin Yu.G., Khruschev V.V., Bosinger T., Kangas J., Prikner K. Observations of IAR spectral resonance structures at a large triangle of geophysical observatories. Proc. XXVI Annual Seminar"Physics of Auroral Phenomena”. Apatity, 2003, pp. 123-126.

Prikner K., Vagner V. Numerical modeling of the ionospheric filtration of an ULF micropulsation signal. Studia Geophysica et Geodaetica. 1983, vol. 27, pp. 173-190.

Prikner K., Vagner V. The ionosphere as an Alfvén resonator in the Pc1 micropulsation range. Studia Geophysica et Geodaetica. 1990, vol. 34, pp. 342-361.

Prikner K., Vagner V. Numerical solution to the problem of ionospheric filtration of ULF waves in the Pc1 range. The total wave field inside the ionospheric transition layer. Studia Geophysica et Geodaetica. 1991, vol. 35, pp. 90-99.

Prikner K., Mursula K., Feygin F.Z., Kangas J., Kerttula R., Pikkarainen T., Pokhotelov O.A., Vagner V. Non-stationary Alfvén resonator: vertical profiles of wave characteristics. J. Atmosph. Solar-Ter. Phys. 2000, vol. 62, pp. 311-322.

Prikner K., Mursula K., Kangas J., Feygin F.Z. Ionospheric Alfvén resonator control over the frequency-variable Pc1 event in Finland on May 14, 1997. Studia Geophysica et Geodaetica. 2001, vol. 45, pp. 363-381.

Semenova N.V., Yahnin A.G. Abrupt change of resonance structure in electromagnetic noise spectrum in hertz range during a substorm. Geomagnetizm i aeronomiya [Geomagnetism and Aeronomy]. 2014, vol. 54, pp. 341-347. (In Russian).

Yahnin A.G., Semenova N.V., Ostapenko A.A., Kangas J., Manninen J., Turunen T. Morphology of the spectral resonance structure of the electromagnetic background noise in the range of $0.1-4 \mathrm{~Hz}$ at L=5.2. Ann. Geophysicae. 2003, vol. 21, pp. 779-786.

How to cite this article

Prikner K., Feygin F.Z., Khabazin Yu.G. Research into connection between spectral resonance structures and harmonics of ionospheric Alfvén resonator. Solar-Terrestrial Physics. 2018. vol. 4, iss. 3, pp. 43-45. DOI: 10.12737/stp-43201806 